\title{
When trainees reach competency in performing endoscopic ultrasound: a systematic review
}

\section{(웅 $\odot$}

\author{
Authors \\ Neal Shahidi, George Ou, Eric Lam, Robert Enns, Jennifer Telford \\ Institutions \\ St. Paul's Hospital, Division of Gastroenterology, Department of \\ Medicine, University of British Columbia, Vancouver, BC, Canada
}

submitted 28.8.2016

accepted after revision $\quad 23.12 .2016$

Bibliography

DOI http://dx.doi.org/10.1055/s-0043-100507 |

Endoscopy International Open 2017; 05: E239-E243

(C) Georg Thieme Verlag KG Stuttgart · New York

ISSN 2364-3722

Corresponding author

Jennifer Telford, MD, Division of Gastroenterology, St. Paul's

Hospital, University of British Columbia, 770-1190 Hornby Street,

Vancouver, BC, Canada

Fax: +1-604-689-2004

jtelford@telus.net

\section{ABSTRACT}

Background/Study aim The American Society for Gastrointestinal Endoscopy (ASGE) recommends that trainees complete 150 endoscopic ultrasound (EUS) procedures before assessing competency.
However, this recommendation is largely based on limited evidence and expert opinion. With new evidence suggesting that this historical threshold is underestimating training requirements, we evaluated the learning curve for achieving competency in EUS.

Patients/Materials and methods Two investigators independently searched MEDLINE for full-text citations assessing the learning curve for achieving competency in EUS in the period 1946 to 25 March 2016. A learning curve was defined as either a tabulated or graphic representation of competency as a function of increasing EUS experience.

Results Eight studies assessing 28 trainees and 7051 EUS procedures were included. When stratifying studies based on procedural indication: three studies assessed competency in evaluating mucosal lesions, three studies assessed competency in EUS fine-needle aspiration (EUS-FNA), and two studies assessed comprehensive competency. Among studies assessing mucosal lesion T-staging accuracy, competency was achieved by 65 to 231 procedures. Among studies assessing EUS-FNA, competency was achieved by 30 to 40 procedures. Among the two studies assessing comprehensive competency in EUS, competency was not achieved in either study across all trainees. Only four of 17 trainees reached competency by 225 to 295 EUS procedures.

Conclusion As EUS competency assessment has evolved to more closely reflect independent clinical practice, the number of procedures required to achieve competency has risen well above ASGE recommendations. Advanced endoscopy training programs and specialty societies need to re-assess the structure of EUS training.

\section{Introduction}

Endoscopic ultrasound (EUS) has emerged as a core diagnostic modality for evaluation and tissue acquisition in gastrointestinal disease [1]. With this comes a critical need for a better understanding of the learning curve to achieve competency in EUS, highlighted by the increasing emphasis on competencybased medical education [2,3]. Unfortunately, due to the current lack of clarity for competency-based assessment in EUS, training programs are still dependent on procedural thresholds to help ensure their trainees achieve competency. This is further complicated by societies [4,5] having to rely on limited data and expert opinion to estimate procedural thresholds with the American Society for Gastrointestinal Endoscopy (ASGE) recommending 150 supervised EUS procedures before competency should be assessed, 75 of which must evaluate the pancreatobiliary system and 50 must incorporate EUS fineneedle aspiration (EUS-FNA) [4].

Recently, systematic reviews for both colonoscopy [6] and endoscopic retrograde cholangiopancreatography (ERCP) [7] have directly questioned their procedural thresholds. Moreover, with new evidence $[8,9]$ suggesting that the current recommendations for EUS also underestimate the volume of procedures required to achieve competency, we sought to systematically evaluate the learning curve for EUS. The goal of this review is to delineate appropriate procedural thresholds thus enabling policymakers in the creation of uniform and objective EUS training guidelines.

\section{Patients/Materials and methods}

This systematic review was completed in accordance with the meta-analysis of observational studies in epidemiology (MOOSE) recommendations [10].

\section{Search strategy}

For the period 1946 to 25 March 2016, two authors (NS and $\mathrm{GO}$ ) independently searched MEDLINE using the following search strategy: ("endosonography [MeSH]" OR "endosonography” OR “endoscopic ultrasound” OR “EUS”) AND (“trainee” OR 
"training" OR "resident" OR "fellow*" OR "competenc*" OR "learning curve"). Subsequently, to evaluate the gray literature, the same authors independently searched the bibliographies of included studies, relevant guidelines, and pertinent review articles for further potential citations.

\section{Study selection}

Full-text citations, published in the English language and which assessed the learning curve in performing EUS, were considered for inclusion. A learning curve was defined as either a tabulated or graphic representation of competency as a function of increasing EUS experience. A minimum of two data points along the learning curve were required. Given the diversity of indications for EUS, quantifying trainee success rate for a specific component of the procedure such as cecal intubation rate for colonoscopy [6] or cannulation rate for ERCP [7] was not mandated for study inclusion.

Citations were subsequently independently assessed for potential exclusion under the following criteria: 1) not published in English; 2) only published as an abstract; 3) review articles, letters, commentaries, editorials or book chapters; 4) lacked trainee-specific or EUS-specific outcomes; 5) assessed non-gastroenterology or non-surgery-based trainees; 6) did not quantify a learning curve as defined above or trainee position along the learning curve was not clear; 7) only the initial aspect of the learning curve was assessed, which was set at $<25$ EUS procedures; 8) solely used simulation models to quantify the learning curve; 9) focused on the assessment of trans-rectal ultrasound (TRUS), endo-rectal ultrasound (ERUS) or colorectal EUS; 10) focused on the assessment of advanced diagnostic modalities (e.g. elastography, endomicroscopy), interventional EUS or cytopathology training. For the purpose of this review, citations in which participating endosonographers who had completed EUS training but were learning EUS-FNA were deemed acceptable for study inclusion. Authors were contacted if further information was believed to potentially affect study inclusion.

\section{Data extraction}

Citations selected for study inclusion subsequently underwent independent data extraction by two authors (NS and GO), with variables including: 1) year of publication; 2) country of origin; $3)$ trainee description including previous endoscopic experience; 4) structure of advanced endoscopy training; 5) EUS case description; 6) trainee's involvement during EUS; 7) EUS competency outcomes. Data was finalized through consensus between the two authors with disagreement at any stage of the systematic review process being resolved by involving a third study author (JT).

\section{Outcomes and analysis}

Our outcome of interest was the number of procedures required to achieve competency in EUS. Estimates were extracted as they were expressed within their respective citation, using their respective marker of competency. When a citation assessed multiple definitions of competency or provided multiple estimates for when competency was achieved, for the purpose of

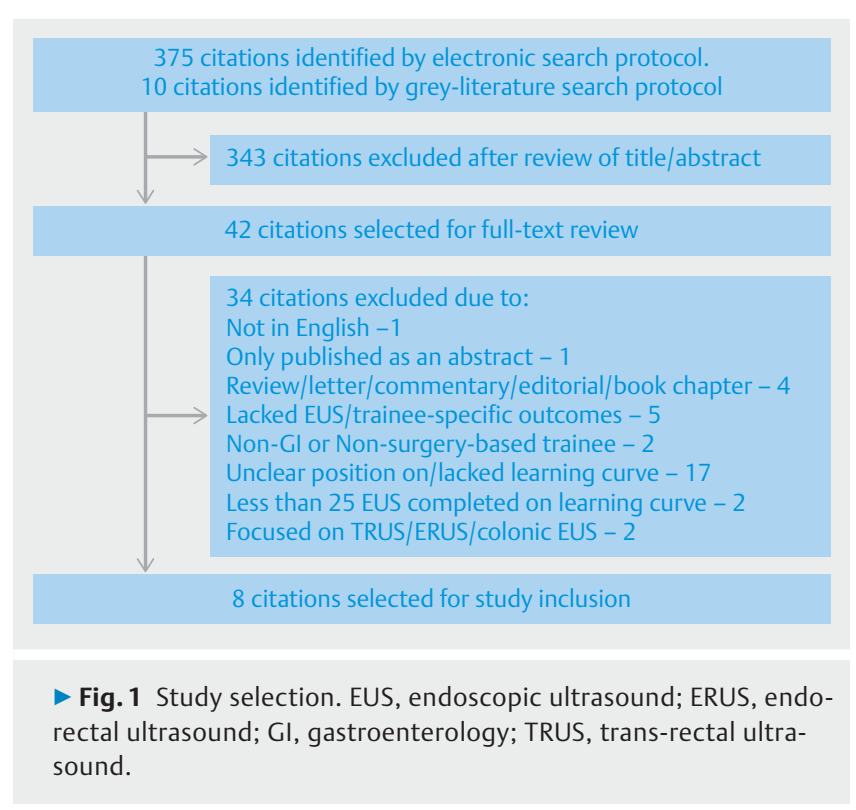

this review, what we perceived to be the primary definition/estimate was used to define when competency was achieved. Citations were subsequently stratified based on site/lesion as per the ASGE credentialing guidelines [4].

Descriptive statistics were used to present study findings as meta-analysis was thought to be inappropriate at this time due to notable heterogeneity.

\section{Results}

\section{Search results and study description}

In total, 385 citations were identified by electronic and greyliterature searches, of which 42 underwent full-text review (> Fig.1). Ultimately, eight studies [8,9,11-16] assessing 28 trainees and 7051 EUS procedures were included ( $\vee$ Table 1 ), and 34 full-text citations were excluded as described in $\mathbf{F i g .}$ 1. Three studies $[8,9,12]$ were prospective in study design with seven studies assessing gastroenterology-based trainees $[8,9,11-14,16]$ and one study [15] assessing a surgery-based trainee. Upon stratifying included studies, three $[11,15,16]$ assessed competency in mucosal lesion evaluation, three [12-14] assessed competency in EUS-FNA, and two [8,9] assessed comprehensive competency ( $\triangleright$ Table 2 ).

\section{Competency assessment: mucosal lesions}

Three studies $[11,15,16]$, including six trainees performing 896 EUS procedures, assessed competency in mucosal lesion evaluation. All three studies focused on T-staging accuracy of esophageal and/or gastric cancer. Only one study [11] clearly defined competency, based on staff T-staging accuracy, whereas the other two studies $[15,16]$ defined a threshold number of required procedures based on a statistically significant difference in trainee performance along the learning curve. It was deemed that competency was achieved across all trainees over a range of 65 to 231 EUS procedures. 
- Table1 Description of included studies.

\begin{tabular}{|l|l|l|l|l|c|}
\hline Study & Year & Country & Study design & Trainee background & \# Trainees \\
\hline Park et al. [11] & 2015 & Republic of Korea & Retrospective & Gastroenterology & 4 \\
\hline Wani et al. [9] & 2015 & USA & Prospective & Gastroenterology & 12 \\
\hline Wani et al. [8] & 2013 & USA & Prospective & Gastroenterology & 5 \\
\hline Cote et al. [12] & 2011 & USA & Prospective & Gastroenterology & 3 \\
\hline Nayar et al. [13] & 2011 & UK & Retrospective & Gastroenterology & 1 \\
\hline Mertz and Gautam [14] & 2004 & USA & Retrospective & Gastroenterology & 1 \\
\hline Schlick et al. [15] & 1999 & Germany & Retrospective & Surgery & 112 \\
\hline Fockens et al. [16] & 1996 & The Netherlands & Retrospective & Gastroenterology & 1 \\
\hline
\end{tabular}

- Table 2 Competency outcomes of included studies.

\begin{tabular}{|c|c|c|}
\hline Study & Marker of competency & Outcomes \\
\hline \multicolumn{3}{|c|}{ Mucosal competency assessment } \\
\hline Park et al. [11] & Gastric cancer T-staging accuracy & Competency achieved @ 65 EUS examinations \\
\hline Schlick et al. [15] & $\begin{array}{l}\text { Esophageal or gastric cardia cancer T-staging } \\
\text { accuracy }\end{array}$ & Competency achieved@ 75 to 105 EUS examinations \\
\hline Fockens et al. [16] & Esophageal cancer T-staging accuracy & Competency achieved @ 101 to 231 EUS examinations \\
\hline \multicolumn{3}{|c|}{ EUS-FNA competency assessment } \\
\hline Cote et al. [12] & Adequacy of EUS-FNA specimens & $\begin{array}{l}\text { Competency achievement not specified. The proportion of } \\
\text { adequate specimens did not change along the learning curve }\end{array}$ \\
\hline Nayar et al. [13] & Pancreatic EUS-FNA diagnostic accuracy & Competency achieved @ 30 EUS examinations \\
\hline Mertz and Gautam [14] & Pancreatic EUS-FNA sensitivity & Competency achieved @ 30 to 40 EUS examinations \\
\hline \multicolumn{3}{|c|}{ Comprehensive competency assessment } \\
\hline Wani et al. [9] & $\begin{array}{l}\text { Median score of "no assistance" across assessed } \\
\text { items on EUS evaluation tool }\end{array}$ & $\begin{array}{l}\text { Competency not achieved across all trainees; } 2 / 12 \text { trainees } \\
\text { achieved competency @ } 225 \text { to } 245 \text { EUS examinations }\end{array}$ \\
\hline Wani et al. [8] & $\begin{array}{l}\text { Median score of "no assistance" across assessed } \\
\text { items on EUS evaluation tool }\end{array}$ & $\begin{array}{l}\text { Competency not achieved across all trainees; } 2 / 5 \text { trainees achieved } \\
\text { competency @ } 255 \text { to } 295 \text { EUS examinations }\end{array}$ \\
\hline
\end{tabular}

\section{Competency assessment: EUS-FNA}

Three studies [12 - 14], including five trainees and 486 EUS procedures, evaluated competency in performing EUS-FNA. Two studies $[13,14]$ focused on pancreatic EUS-FNA. Competency was clearly defined in one study [13] as a diagnostic accuracy of $>70 \%$. Of the two remaining studies, one study [14] defined a threshold number of required procedures based on a statistically significant difference in trainee performance along the learning curve. The focus of the other study [12] was to assess the feasibility of concurrent EUS-FNA training alongside EUS training; competency was not clearly defined. Of the two studies $[13,14]$ in which competency was deemed to have been achieved, this occurred over a range of 30 to 40 EUS-FNA procedures.

\section{Competency assessment: comprehensive competency}

Two studies [8,9], including 17 trainees and 5669 EUS procedures, evaluated comprehensive competency. For both studies, a successful procedure was defined by a median score of 1 (no assistance) on a standardized EUS assessment tool; this included when applicable: 1) identifying the lesion of interest; 2) TNM staging; 3) sub-epithelial wall-layer characterization; 4) EUSFNA. Using cumulative sum (CUSUM) analysis, acceptable and unacceptable failure rates of $10 \%$ and $20 \%$, respectively, were used to define competency. Competency was not achieved in either study across all trainees; only four of 17 trainees reached competency between 225 and 295 EUS procedures. 


\section{Discussion}

As EUS has become an integral tool in the armamentarium of the advanced endoscopist, increasing emphasis has been placed on refining EUS training. Naturally, similar to the maturation of competency assessment for colonoscopy [6] and ERCP [7], questions have been raised concerning the adequacy of current recommendations [4] with regard to the number of EUS procedures required before assessing competency. Furthermore, recent data indicate that, even with the current recommendations in mind, training programs are providing insufficient EUS procedural volumes [17]. After these findings, studies began to emerge re-evaluating procedural thresholds for achieving competency in EUS [8,9]. Alongside this, a systematic review has been published evaluating training and competency assessment in endoscopy, including EUS [18]. However, this review included only two studies $[8,19]$ assessing learning curves and procedural thresholds in EUS and, given the emergence of a landmark study in EUS competency [9], we felt that a systematic review focusing on EUS competency assessment was warranted. With the above in mind, our systematic review highlights that, as competency assessment in EUS has evolved and has begun to assess comprehensive competency, the number of procedures required to achieve competency in EUS remains unclear but has clearly risen above current ASGE recommendations. Given these findings and in the wake of competency-based medical education, advanced endoscopy training programs and specialty societies need to re-evaluate the current structure of EUS training.

Historically, colonoscopy $[20,21]$, ERCP $[22,23]$, and EUS have shared a similar evolution with initial recommendations for the number of procedures trainees should perform before assessing competency being largely based on limited data and expert opinion. Although this appears to be the nature of procedural development, with studies now evaluating comprehensive competency, guidelines must adapt and reflect the new era of EUS competency assessment. Traditionally, while the scope of EUS practice may have been more focused on a specific indication for EUS, we believe current trainees must at least be able to perform mucosal tumor evaluation, submucosal abnormality evaluation, pancreatobiliary evaluation, and EUS-FNA. Therefore, the concept of independent comprehensive competency should be the new standard for defining competency in EUS. Until competency-based assessment in EUS can be delineated, we agree with Wani et al. [9] that at least 225 EUS procedures should be performed before assessing competency. It is important to note though that this was the earliest that a trainee achieved "comprehensive competency" and that only four of 17 trainees achieved competency by 225 to 295 EUS procedures with an average of approximately 330 EUS procedures per trainee. Therefore, with the threshold for comprehensive competency remaining unclear, advanced endoscopy training programs should expect that most trainees will not reach competency at either 225 EUS procedures or 330 EUS procedures and need to modify their programs accordingly.

Although the evolution of the definition of competency in EUS may mirror that of colonoscopy and ERCP, the assessment of EUS competency is much more difficult. Both colonoscopy and ERCP have well-established quality indicators such as cecal intubation rate [24] and native papilla deep cannulation [25], which have been used to assess trainee competency. Unfortunately, EUS has no such quality indicator [26]. This is in part due to the variety of indications for EUS, thus making it difficult to identify a universal EUS procedural component across all EUS indications; and while EUS-FNA sample adequacy rates, EUSFNA sensitivity, and adverse events incidence rates could potentially be used as markers of competency, limiting factors have prevented their adoption [27]. Concerning EUS-FNA sample adequacy rates and EUS-FNA sensitivity, as described above, these only pertain to EUS-FNA. Moreover, none of these can be readily quantified during or immediately after the procedure which limits their utility. Given the above, we commend Wani and colleagues $[8,9]$ for the creation of an objective standardized assessment tool in EUS. Through the incorporation of cognitive evaluation, therapeutic EUS, as well as delineating a universal approach to station/procedural component scoring, it is our belief that this tool carries with it the potential to emerge as a means for objectively quantifying comprehensive competency.

Our study has a number of limitations. Most notably, due to multiple sources of heterogeneity including study methodology, the definition of competency in EUS, trainees' previous endoscopic experience including duodenoscope experience, EUS training environment, and the indication for EUS, we felt that meta-analysis was not appropriate at this time. Moreover, quality assessment of included studies was not performed, to remain consistent with our previous reviews on competency assessment in colonoscopy [6] and ERCP [7]. Our exclusion criteria were relatively restrictive, which potentially led to publication bias due to the exclusion of non-English publications, colorectal EUS, and interventional EUS. Alongside this, given our focus on gastroenterology and surgery trainees, we excluded studies evaluating competency in EUS for lung cancer amongst pulmonary trainees $[28,29]$. Of note, recent recommendations from the European Society of Gastrointestinal Endoscopy (ESGE), in conjunction with the European Respiratory Society (ERS) and the European Society of Thoracic Surgeons (ESTS) have been published addressing this specific indication [30]. Lastly, we included studies assessing the initial methods for training in EUS [14-16] which no longer reflect the current structure of EUS training.

In conclusion, our systematic review highlights that as EUS competency assessment has evolved to more closely reflect independent clinical practice, the number of EUS procedures required to achieve competency remains unclear but has clearly risen above current ASGE recommendations. Further research is required to establish standardized definitions and means of assessing comprehensive competency and all EUS indications, thus facilitating individualized competency assessment dependent on the trainee's future breadth of practice. Moreover, as EUS training continues to incorporate therapeutic EUS, the definition of comprehensive competency will need to evolve alongside. Moving forward, advanced endoscopy training programs must embrace more conservative procedural thresholds 
for achieving competency in EUS and provide adequate procedural volumes to their trainees. However, it is critical to understand that trainees achieve competency over a range of EUS procedures. Therefore, each trainee requires individualized assessment, to ensure competency is achieved before entering independent clinical practice.

\section{Competing interests}

None

\section{References}

[1] Early DS, Ben-Menachem T, Decker GA et al. Appropriate use of GI endoscopy. Gastrointest Endosc 2012; 75: 1127-1131

[2] Boyce WH. Training in endoscopic ultrasonography. Gastrointest Endosc 1996; 43: S12-S15

[3] DiMaio C], Mishra G, McHenry L et al. EUS core curriculum. Gastrointest Endosc 2012; 76: 476-481

[4] Eisen GM, Dominitz JA, Faigel DO et al. Guidelines for credentialing and granting privileges for endoscopic ultrasound. Gastrointest Endosc 2001; 54: $811-814$

[5] Polkowski M, Larghi A, Weynand B et al. Learning, techniques, and complications of endoscopic ultrasound (EUS)-guided sampling in gastroenterology: European Society of Gastrointestinal Endoscopy (ESGE) Technical Guideline. Endoscopy 2012; 44: 190-206

[6] Shahidi N, Ou G, Telford J et al. Establishing the learning curve for achieving competency in performing colonoscopy: a systematic review. Gastrointest Endosc 2014; 80: 410-416

[7] Shahidi N, Ou G, Telford J et al. When trainees reach competency in performing ERCP: a systematic review. Gastrointest Endosc 2015; 81: $1337-1342$

[8] Wani S, Cote GA, Keswani R et al. Learning curves for EUS by using cumulative sum analysis: implications for American Society for Gastrointestinal Endoscopy recommendations for training. Gastrointest Endosc 2013; 77: $558-565$

[9] Wani S, Hall M, Keswani RN et al. Variation in aptitude of trainees in endoscopic ultrasonography, based on cumulative sum analysis. Clin Gastroenterol Hepatol 2015; 13: $1318-1325$

[10] Stroup DF, Berlin JA, Morton SC et al. Meta-analysis of observational studies in epidemiology. JAMA 2000; 283: 2008-2012

[11] Park CH, Park JC, Kim EH et al. Learning curve for EUS in gastric cancer T staging by using cumulative sum analysis. Gastrointest Endosc 2015; 81: 898-905

[12] Cote GA, Hovis CE, Kohlmeier C et al. Training in EUS-guided fine needle aspiration: Safety and diagnostic yield of attending supervised, trainee-directed FNA from the onset of training. Diagn Ther Endosc 2011; 2011: 378540

[13] Nayar M, Joy D, Wadehra V et al. Effect of dedicated and supervised training on achieving competency in EUS-FNA of solid pancreatic lesions. Scand J Gastroenterol 2011; 46: 997-1003
[14] Mertz H, Gautam S. The learning curve for EUS-guided FNA of pancreatic cancer. Gastrointest Endosc 2004; 59: $33-37$

[15] Schlick T, Heintz A, Junginger T. The examiner's learning effect and its influence on the quality of endoscopic ultrasonography in carcinoma of the esophagus and gastric cardia. Surg Endosc 1999; 13: 894-898

[16] Fockens P, Van den Brande JHM, Van Dullemen HM et al. Endosonographic T-staging of esophageal carcinoma: a learning curve. Gastrointest Endosc 1996; 44: $58-62$

[17] Azad JS, Verma D, Kapadia AS et al. Can U.S. GI fellowship programs meet American Society for Gastrointestinal Endoscopy recommendations for training in EUS? A survey of U.S. GI fellowship program directors Gastrointest Endosc 2006; 64: 235-243

[18] Ekkelenkamp VE, Koch AD, de Man RA et al. Training and competence assessment in $\mathrm{Gl}$ endoscopy: a systematic review. Gut 2016; 65: $607-$ 615

[19] Meenan J, Anderson S, Tsang S et al. Training in radial EUS: what is the best approach and is there a role for the nurse endoscopists? Endoscopy 2003; 35: $1020-1023$

[20] Wigton RA, Blank LL, Monsur $\mathrm{H}$ et al. Procedural skills of practicing gastroenterologists. A national survey of 700 members of the American College of Physicians. Ann Intern Med 1990; 113: 540 - 546

[21] American Society for Gastrointestinal Endoscopy. Principles of training in gastrointestinal endoscopy. Gastrointest Endosc 1999; 49: $845-854$

[22] Wigton RS, Vennes JA. Clinical competence in diagnostic endoscopic retrograde cholangiopancreatography. Ann Intern Med 1988; 108: $142-144$

[23] Cockeram A. Canadian Association of Gastroenterology Practice Guideline for clinical competency in diagnostic and therapeutic endoscopic retrograde cholangiopancreatography. Can J Gastroenterol 1997; 11: 539-542

[24] Rex DK, Schoenfeld PS, Cohen J et al. Quality indicators for colonoscopy. Gastrointest Endosc 2015; 81: 31-53

[25] Adler DG, Lieb JG, Cohen J et al. Quality indicators for ERCP. Gastrointest Endosc 2015; 81: 54-66

[26] Wani S, Wallace MB, Cohen J et al. Quality indicators for EUS. Gastrointest Endosc 2015; 81: 67-80

[27] Rosch T. State of the art lecture: Endoscopic ultrasonography: Training and competence. Endoscopy 2006; 38: S69-S72

[28] Annema JT, Bohoslavsky R, Burgers S et al. Implementation of endoscopic ultrasound for lung cancer staging. Gastrointest Endosc 2010; 71: $64-70$

[29] Konge L, Annema J, Vilmann P et al. Transesophageal ultrasonography for lung cancer staging learning curves of pulmonologists. J Thorac Oncol 2013; 8: 1402 - 1408

[30] Vilmann P, Clementsen PF, Colella S et al. Combined endobronchial and oesophageal endosonography for the diagnosis and staging of lung cancer. European Society of Gastrointestinal Endoscopy (ESGE) Guideline, in cooperation with the European Respiratory Society (ERS) and the European Society of Thoracic Surgeons (ESTS). Eur Respir J 2015; 46: $40-60$ 